CELL STRUCTURE AND FUNCTION 9, Suppl., s39-42 (1984)

(C) by Japan Society for Cell Biology

\title{
II-5 Mitochondria
}

\author{
Katsuro Koike \\ Department of Gene Research, Cancer Institute, JFCR, Toshima-ku, \\ Tokyo 170, Japan
}

Although it is difficult to present a short review of all the mitochondrial researchs performed in the past several years in Japan and to cover all of the Japanese scientists engaged in this research, I have outlined major studies done on the mitochondrial genetic system. It is clear that the powerful techniques of base-sequence analysis and gene recombination have led us to a point of unlimited possibilities, although they have not enabled us to study all the relevant questions that have been raised. In general, much interesting data have been accumulated. The first part of this overview deals with studies of the genomic structure developed by recombinant DNA technology and the nucleotide sequencing technique. The second and third parts summarize the biochemical approachs used for determining the mechanisms that lead to the biogenesis of mitochondria.

\section{Mitochondrial gene organization}

Rapid progress has been made in mammalian mtDNA studies. The complete structure of human, mouse and rat mtDNAs have become known in the past few years. And, it has been convincingly shown that for yeast mtDNA the mitochondrial genome contains genetic information comparable to that found in mammalian mtDNAs.

The primary aim of this type of research is to determine how genetic functions have been conserved in mammalian mitochondria in such a small content of genetic information. The results of studies by Koike and his colleagues (Kobayashi et al., 1981; Koike et al., 1982) with the rat mitochondrial genome have given us important clues. The data for base sequencing indicates that the gene organizations in the rat and other mammalian mtDNAs are fully consistent with each other. Rat genes contain sequences homologous to other mammalian and yeast genes, which is interesting in view of the homology showing that the $3^{\prime}$-end of $12 S$ rat mitochondrial rRNA is a consensus sequence that of other mitochondrial rRNA and even that of E. coli rRNA. The bases of rat mtDNA are most efficiently used for coding, in which a number of genes are buttjointed.

Although it has been hypothesized that for mammalian mtDNAs the stop codons of the messenger RNAs for many genes are brought about through a posttranscriptional event, such as cutting adjacent mRNAs and tRNAs with concomitant polyadenylation at the $3^{\prime}$-end of the mRNA, the UAA stop signal was unambiguously present at the $3^{\prime}$-end of rat apocytochrome $b$ gene. In addition to genes for known polypeptides, a number of as yet unidentified reading frames (URFs) also have been found in rat mtDNA, these potentially code for hydrophobic membrane-type proteins. Data for human, bovine, and mouse mtDNAs are all in good agreement with the data reported for rat mtDNA. 
Organization of the mitochondrial genome in lower eukaryotes is characterized by the presence of mosaic genes. This split gene structure was first found in the large rRNA of $\omega$ strains of Saccharomyces cerevisiae. Other structural genes with mosaic structures, the cytochrome $\mathrm{b}$ gene and the gene for subunit I of the cytochrome c oxidase, in Saccharomyces cerevisiae, also have been characterized. In particular, a number of papers have reported on the exon-intron structure of the apocytochrome $b$ gene; unfortunately, however, in this area no significant contribution has yet been made by Japanese researchers.

In "short" strains there are 3 exons and 2 introns; but in "long" strains there are 5 exons and 4 introns. In the latter strains the exons are $\alpha, \beta, \gamma, \delta$, and $\varepsilon$, and the introns are $\mathrm{I} \alpha \beta, \mathrm{I} \beta \gamma, \mathrm{I} \gamma \delta$, and $\mathrm{I} \delta \varepsilon$. The most interesting feature is the presence and expression of reading frames in some of the introns. For box 3, in particular, the genetic and biochemical data complement each other. The intron-gene product is the so-called maturase. In the first step, the 10S circular RNA is released after which the $\alpha$ exon links to the reading frame in $\mathrm{I} \alpha \beta$. This leads to the production of the maturase responsible for the further processing and linkage of the $\alpha$ to the $\beta$ exon. The experiments reported are excellent examples of how posttranscriptional modification may occur, also, they show how nucleocytoplasmic and mitochondrial activities may be interlinked.

The petite mutants of yeast have been studied by many researchers. The petite mtDNAs, originally used in deletion mapping for the estimation of the gene order in yeast mtDNA, have now been successfully introduced in studies of sequences that can function as a replicator. The initial approach took advantage of hypersuppressive petites, and their DNAs were isolated and sequenced. Common sequences were found on various segments of hypersuppressive petite mtDNA. These sequences(ori) were designated as ori 1 to ori 8 for the origin of replication in wild type mtDNA.

Petites with lower suppressiveness also can replicate in yeast cells, but they possess segments of mtDNA that differ from those of wild type mtDNA. The diverse occurrences of these sequences suggest that sequences other than the ori sequences can function as replicators. Wakabayashi and his colleagues (Wakabayashi, 1980; Mabuchi, Nishikawa and Wakabayashi, 1983) studied the high segregation of oligomycin-resistant petites and cloned two autonomously replicating sequences from a short segment of the mtDNA of an oligomycin-resistant yeast into a vector pYleu12 constructed from yeast LEU2 gene and pBR322. These plasmids, pYmt4 and $\mathrm{pYmtl}$, had frequencies for the transformation of yeast as high as that of $\mathrm{YEpB}$, containing a replication origin of $2 \mu$ DNA. They also found that these sequences were located in intergenic regions.

Apart from the genes for well known respiratory enzyme components and for the maturases described above, the information about mitochondrial genes coding for polypeptides is insufficient. This area certainly deserves and needs further investigation. The URFs of mammalian mtDNA already have been described as potential genes. For yeast there are few unidentified reading frames, but the polypeptides deduced from the sequences show somewhat odd amino acid compositions.

\section{Mitochondrial replication, transcription and translation}

Mitochondria grow and divide in many organisms, but the mechanism of mtDNA replication is not well understood. For the lower eukaryotes, in particular, there is little information. The mitochondria of Physarum have unique advantages for use in the study of the mechanisms of the distribution of mtDNA during the period of 
mitochondrial division because mitochondrial division is semisynchronized in a plasmodium, and the mitochondrion contains an electron dense mt-nucleus. The mitochondrion of Physarum contains about 60 molecules of mtDNA, one of which is linear and approximately $12 \mu \mathrm{m}$ long. Kuroiwa and his coleagues (Kuroiwa, Kawano and Hizume, 1977; Kawano and Kuroiwa, 1979) have investigated how the 60 molecules of this mtDNA behave during mitochondrial division and using a lightmicroscopy autoradiographic technique, they have presented direct evidence that the individual DNA molecule separates randomly, but equally, into two daughter mitochondria. Their electron-microscopic autoradiograms of dumbbell-shaped and daughter matochondria also show that the equal distribution of the mt-nucleus.

In animal systems, the origin of replication has been characterized in some detail by Koike and his colleagues (Tanaka and Koike, 1978; Sekiya et al., 1980; Yaginuma et al., 1982). Mammalian mtDNA contains a unique DNA sequence (the D-loop region) that has been defined as a requirement for the initiation of unidirectional DNA replication. Unlike the rest of the genome, this region of about $900 \mathrm{bp}$ varies greatly in sequence and size among mammalian species except for several small blocks of homology. Functionally, the homologous portions are separable into two areas; the upstream and downstream regions from the $5^{\prime}$-end of the D-loop DNA $\left(\mathrm{O}_{\mathrm{H}}\right)$. Of the DNA-synthesizing enzymes, DNA polymerase $\gamma$ seems to be the active enzyme in mitochondria although the functioning of some other DNA polymerase cannot be excluded.

Some data on mitochondrial topoisomerases have been presented, but the picture is far from complete yet. The same is true for studies on transcription, but reasonable progress has been made in the characterization of rat mitochondrial RNA polymerase which is specific for the D-loop region of mtDNA in the supercoiled structure.

Notable progress has been made with respect to the mechanism of translation. First, the divergence of codon usage has been found in mitochondria. The termination codon, UAG, in the "universal" code appears to be tryptophan in mitochondria. The isoleucine codon, AUA, also is believed to be a start codon in mitochondria. With respect to the mitochondrial tRNA structure the conclusion is that in mammals the cloverleaf structure is less concerved. The structure that deviates greatly from the structures of classical tRNA has been found in the sequence done by Watanabe and his colleagues (Ueda, Watanabe and Ohta, 1983a, b) of the bovine tRNA $A_{A G Y}^{S e r}$ structure, in which the T-stem and T-loop are present but the D-arm is not.

In view of the general belief that mitochondrial ribosomes do not contain a $5 \mathrm{~S}$ rRNA, there is a serious question about the mechanism of interaction between tRNAs and ribosomes because mammalian and other animal mitochondrial tRNAs show deviating structures. Up to now, no one has successfully established a reconstituted system for mitochondrial protein synthesis in vitro using mitochondrial apparatus such as mitochondrial ribosomes, tRNAs and protein factors.

\section{Regulatory and developmental aspects of mitochondrial biogenesis}

Various experimental systems have been used to study regulation of the biogenesis of mitochondria during growth and development. The genetic approach is paticularly popular and useful. The results of such studies, however, are mainly descriptive and do not readily lead to an understanding of the mechanisms that regulate the interactions of the nucleocytoplasmic and mitochondrial genetic systems. 
In this area our knowledge shows more gaps than facts; nevertheless, there have been several interesting achievements. The investigations of Morita and Miura (Miura et al., 1982; Morita et al., 1982), who used inhibitors for the transfer of a precursor of ornithine transcarbamylase into mitochondria, have led to a model in which a number of, though not all, cytoplasmically synthesized mitochondrial proteins are made as precursors. Furthermore, there is an energy-dependent transfer of these proteins into the mitochondria. The presence and activity of a specific mitochondrial protease is especially interesting in this context. The hypothesis that there are specific recognition points, or receptors, on the mitochondrial membrane for cytoplasmically synthesized mitochondrial proteins is attractive, but further investigations are needed.

Translational factors as well as ribosomal proteins in mitochondria first are encoded by nuclear genes, then synthesized in the cytoplasmic fractions and transported to the mitochondria. The organization of the nuclear genes for the mitochondrial translational apparatus, their expression and the incorporation of this protein into mitochondria are not well understood. Very recently, Kaziro and his colleagues (Nagata et al., 1983) reported molecular cloning of the yeast mitochondrial EF-Tu gene (tuf M). The nucldotide sequence, as well as the deduced amino acid sequence of the tuf M gene, indicates that a nuclear-coded mitochondrial EF-Tu protein is synthesized as a precursor in the cytoplasm then is transported to the mitochondria by use of the first 20 amino acid residues of a possible signal sequence facing the mitochondrial matrix.

\section{REFERENCES}

Kawano, S. and Kuroiwa, T. (1979). Cell Struct. Funct. 4, 99-108.

Kobayashi, M., Seki, T., Yaginuma, K. and Koike, K. (1981). Gene 16, 297-307.

Koike, K., Kobayashi, M., Yaginuma, K., Taira, M., Yoshida, E. and Imai, M. (1982). Gene 20, 177-185.

Kuroiwa, T., Kawano, S. and Hizume, M. (1977). J. Cell Biol. 72, 687-694.

Mabuchi, T., Nishikawa, S. and Wakabayashi, K. (1983). J. Biochem., in press.

Miura, S., Mori, M., Amaya, Y. and Tatibana, M. (1982). Eur. J. Biochem. 122, 641-647.

Morita, T., Miura, S., Mori, M. and Tatibana, M. (1982). Eur. J. Biochem. 122, 501-509.

Nagata, S., Yokota, Y., Naito, A. and Kaziro, Y. (1983). Proc. Natl. Acad. Sci. USA 80, 6192-6196.

Sekiya, T., Kobayashi, M., Seki, T. and Koike, K. (1980). Gene 11, 53-62.

Tanaka, S. and Koike, K. (1978). Biochem. Biophys. Res. Commun. 81, 791-797.

Ueda, T., Watanabe, K. and Ohta, T. (1983a). Nucleic Acids Res. Symp. Series, No. 12, 141-144.

Ueda, T., Watanabe, K. and Ohta, T. (1983b). Proc. J. Acad., in press.

WakABAyashi, K. (1978). Mol. Gen. Gent. 159, 229-238.

Yaginuma, K., Kobayashi, M., Taira, M. and Koike, K. (1982). Nucleic Acids. Res. 10, 75317542. 